\title{
The diagnostic value of proteoglycan 4 in the differentiation of stable and exacerbation periods in chronic obstructive pulmonary disease: a preliminary study.
}

\author{
Oguz Eroglu ${ }^{*}$, Nermin Dindar Badem², Ayse Baccioglu ${ }^{3}$ \\ ${ }^{1}$ Department of Emergency Medicine, Faculty of Medicine, Kirikkale University, Kirikkale, Turkey \\ ${ }^{2}$ Department of Medical Biochemistry, Faculty of Medicine, Kirikkale University, Kirikkale, Turkey \\ ${ }^{3}$ Divison of Immunology and Allergy Disesases, Department of Pulmonary Diseases, Faculty of Medicine, Kirikkale \\ University, Kirikkale, Turkey
}

\begin{abstract}
Background and aim: Chronic Obstructive Pulmonary Disease (COPD) is characterized by exacerbation and stable periods. Early diagnosis in the exacerbation period of COPD can reduce morbidity and mortality rates. The aim of this study is to investigate the relationship between Proteoglycan 4 (PRG4) and other parameters, including Procalcitonin (PCT) and C-reactive protein (CRP) levels and White Blood Cell (WBC) count, in the exacerbation period.

Materials and methods: This preliminary study was conducted on patients admitted to the Emergency Department and Chest Diseases Department in University Medical Faculty Hospital. Patient demographics, spirometric measurements, clinical scoring used for COPD classification and laboratory test results were recorded for each patient. The final diagnosis of COPD was made by pulmonary physicians.

Results: A total of 78 patients (38 in exacerbation and 40 in stable period) with COPD were included in the study. PRG4 levels of patients in the exacerbation period $(183.7 \pm 9.4 \mathrm{ng} / \mathrm{ml})$ were significantly higher than those of patients in the stable period $(177.1 \pm 15.9 \mathrm{ng} / \mathrm{ml} ; \mathrm{p}=0.034)$. The sensitivity of PRG4 (88.9\%) was equal to that of PCT (88.9\%) and higher than that of CRP (86.1\%) and WBC (80.6\%); the specificity of PRG4 $(50 \%)$ was higher than that of the other parameters $(33.3 \%, 43.0 \%$, and $40.0 \%$, respectively).

Conclusion: PRG4 can be used for discriminating between the exacerbation and stable periods of COPD and is superior in diagnosing the exacerbation period compared with other laboratory parameters.
\end{abstract}

Keywords: Chronic obstructive pulmonary disease, Exacerbations, Proteoglycan-4.

Accepted on March 28, 2018

\section{Introduction}

Chronic Obstructive Pulmonary Disease (COPD) is a progressive disease characterized by stable or exacerbation periods [1]. Although its pathophysiology has not yet been fully elucidated, it has been reported that circulating levels of molecules such as TNF- $\alpha$, IL- $1 \beta$, IL-6, and IL-8 that are produced in the process of developing systemic inflammation tend to increase, which in turn trigger acute-phase reactants such as C-Reactive Protein (CRP), fibrinogen, serum amyloid $\mathrm{A}$, and protein D. This continues in the course of chronic systemic inflammation of the disease, and becomes even more pronounced during the exacerbation period [1-4]. Increased mortality and morbidity rates can be reduced by rapid and accurate diagnosis of patients in the exacerbation period $[1,2]$. Spirometric measurements, through which respiratory functions are evaluated, are used in the diagnosis and follow- up of the disease [1]. In addition, immediate spirometric measurements cannot be taken for patients presenting with COPD in the exacerbation period to the Emergency Department; in order to make a definitive diagnosis of the exacerbation period, detailed clinical evaluation, various laboratory tests, and radiodiagnostic examination should be performed. Although many factors play a role in the development of the exacerbation period in patients with COPD, the most important factor is infection [1,2]. Infections coupled with chronic inflammatory process present in patients with COPD may result in misleading laboratory results. This may lead to certain doubts about diagnosis, delay of diagnosis, and request for additional diagnostic examinations, which in turn causes loss of time and increase in expenditure [1,2].

Proteoglycans are biological molecules produced by eukaryotic cells and have been shown to be associated with various 
pathologies. In various studies, Proteoglycan-4 (PRG4) has been found in several organs such as the lung, heart, liver, bone, and cartilage and has several biological functions such as immunoreactivity, cytoprotection, lubrication, and matrix binding [5-7].

The aim of this study is to investigate whether PRG4 can be used to differentiate exacerbation and stable periods of COPD.

\section{Materials and Methods}

\section{Study design}

This study was approved by the Local Ethics Committee (No. 2016-05/06). This prospective study included patients diagnosed with COPD who were admitted to the Emergency Department and Chest Diseases Department of Kirikkale University Medical Faculty Hospital between May 2016 and May 2017. Patients gave voluntary consent to participate in the study, and the study was conducted in accordance with the principles of the Declaration of Helsinki.

\section{Selection of patients}

This study included patients with exacerbation and stable periods who were previously diagnosed with COPD. The definitive diagnosis and the final decision of the assignment of patients to different groups for evaluation were made by pulmonary physicians.

- Patients in the exacerbation period: Patients who were previously diagnosed with COPD and who were admitted to the Emergency Department with acute onset dyspnea, decreased daily activity, increased sputum volume and changed color, increased cough, high fever, and/or impaired mental functioning were diagnosed to be in the exacerbation period.

- Patients in the stable period: Patients who were previously diagnosed with COPD and who presented to the Chest Diseases Polyclinic for routine control or prescribed with COPD drugs and who had no active complaints were considered to be in the stable period.

Patient demographics (age, gender, Body Mass Index (BMI), smoking status, physical examination findings, COPD duration, and number of attacks per year), spirometric measurements (Global Initiative for Obstructive Lung Disease (GOLD) stage), Modified Medical Research Council (mMRC grade) dyspnea scale, COPD Assessment Test (CAT score) and Combined Assessment of COPD (mortality risk) score, laboratory parameters (CRP, PCT, biochemistry, hemogram, and arterial blood gas results), and PRG4 levels were recorded for each patient.

Patients were excluded if they showed any signs of pulmonary infection or any systemic infection, including any inflammatory disease, which would affect PRG4 levels. Patients with cancer; liver, heart and kidney failure; or any musculoskeletal system disorders that might affect PRG4 levels were also excluded from the study.

\section{Measurement of PRG4 leveI}

Blood samples taken from patients were centrifuged at 4500 $\mathrm{rpm}$ for $10 \mathrm{~min}$ and were stored at $-80^{\circ} \mathrm{C}$. These samples were analysed using the Human PRG4 Elisa kit (Elabscience Biotechnology Co., Ltd, Wuhan, China; Catalog No: E-ELH1675, Lot: AK0016JUL05007). The results are presented as "ng/ml".

\section{Statistical analysis}

Data were analysed using SPSS 23.0 software (Statistical Package for the Social Sciences Inc.; Chicago, IL, USA). The Kolmogorov-Smirnov test was used to evaluate the distribution of data. Average and standard deviation were used in the representation of parametric data; median and Interquartile Range (IQR) were used in the representation of non-parametric data; and number (n) and percentage (\%) were used in the representation of categorical variables. Student's t-test and ANOVA were used for the analysis of parametric data; MannWhitney $U$ test was used for the analysis of non-parametric data; and Pearson's chi-square and Fisher's exact tests were used for the analysis of categorical variables. Spearman's rho correlation test was used to compare quantitative data. ROC curves were used to assess cut-off, sensitivity, and specificity of the data. Results were evaluated in a 95\% Confidence Interval (CI). A value of $\mathrm{p}<0.05$ was considered to be statistically significant.

\section{Results}

A total of 78 patients (38 patients in the exacerbation period and 40 in the stable period) with COPD were included in the study. Ten patients (six in the exacerbation period and four in the stable period) were excluded from the study as their PRG4 levels could not be measured because of hemolysis in the blood samples.

Table 1. Comparison of exacerbation and stable periods in COPD.

\begin{tabular}{|c|c|c|c|}
\hline \multirow[t]{2}{*}{ Data of patients $(n=78)$} & $E P(n=38)$ & $S P(n=40)$ & \multirow[t]{2}{*}{$\mathbf{P}$} \\
\hline & mean \pm SD & mean \pm SD & \\
\hline Age & $65.0 \pm 11.3$ & $65.0 \pm 9.9$ & 0.991 \\
\hline BMI $\left(\mathrm{kg} / \mathrm{m}^{2}\right)$ & $25.9 \pm 5.5$ & $27.6 \pm 5.9$ & 0.206 \\
\hline Smoking time $(y)$ & $27.9 \pm 12.3$ & $26.6 \pm 12.5$ & 0.878 \\
\hline Duration of COPD (y) & $9.3 \pm 4.3$ & $8.4 \pm 4.5$ & 0.743 \\
\hline Number of attacks per year & $9.2 \pm 2.1$ & $7.1 \pm 2.8$ & 0.651 \\
\hline CAT score & $16.5 \pm 14.9$ & $18.0 \pm 8.9$ & 0.756 \\
\hline PRG4 (ng/ml) & $183.7 \pm 9.4$ & $177.1 \pm 15.9$ & $0.034^{*}$ \\
\hline PCT (ng/ml) & $0.192 \pm 0.07$ & $0.07 \pm 0.02$ & 0.073 \\
\hline CRP (mg/l) & $39.7 \pm 9.1$ & $7.8 \pm 3.4$ & $0.034^{\dagger}$ \\
\hline WBC $\left(10^{3} / \mathrm{mm}^{3}\right)$ & $9.5 \pm 5.5$ & $9.6 \pm 5.4$ & 0.139 \\
\hline Neutrophil (\%) & $70.3 \pm 11.1$ & $67.8 \pm 11.2$ & 0.337 \\
\hline
\end{tabular}


The diagnostic value of proteoglycan 4 in the differentiation of stable and exacerbation periods in chronic obstructive pulmonary disease: a preliminary study

\begin{tabular}{llll}
\hline Monocyte (\%) & $6.2 \pm 3.6$ & $11.9 \pm 7.1$ & 0.004 \\
\hline Lymphocyte (\%) & $16.8 \pm 14.4$ & $22.7 \pm 16.2$ & 0.069 \\
\hline Hemoglobin (g/dl) & $14.4 \pm 1.8$ & $14.9 \pm 2.4$ & 0.303 \\
\hline Creatinine (mg/dl) & $0.89 \pm 0.21$ & $0.82 \pm 0.22$ & 0.121 \\
\hline pH (arterial blood gas) & $7.47 \pm 0.1$ & $7.48 \pm 0.1$ & 0.488 \\
\hline Oxygen saturation (\%) & $81.4 \pm 6.2$ & $86.1 \pm 3.4$ & 0.056
\end{tabular}

COPD: Chronic Obstructive Pulmonary Disease; EP: Exacerbation Period; SP: Stable Period; BMI: Body Mass Index; SD: Standard Deviation; PRG4: Proteoglycan-4; PCT: Procalcitonin; CRP: C-Reactive Protein; WBC: White Blood Cell; CAT: COPD Assessment Test; "Student t-test; ${ }^{\dagger}$ Mann-Whitney U test.

The mean age of the patients was $65.0 \pm 10.6 \mathrm{y}$, and $79.5 \%$ were male. The mean age of the patients in the exacerbation period was $65.0 \pm 11.3 \mathrm{y}$ and that of the patients in the stable period was $65.0 \pm 9.9$ y. There was no statistically significant difference between the groups with respect to age $(p=0.991)$. In terms of gender, $84.2 \% \quad(n=32)$ of the patients in the exacerbation period were male, whereas $75 \%(n=30)$ of the patients in the stabilization period were male. There was no statistically significant difference between the groups with respect to gender $(\mathrm{p}=0.314)$.

When patients with COPD in the exacerbation period and those in the stable period were compared, no significant betweengroup difference was found with respect to BMI, smoking time, duration of COPD, number of attacks per year, and CAT score ( $p>0.05$; Table 1$)$. No statistically significant betweengroup difference was found in terms of PCT level, WBC count, neutrophil count, lymphocyte count, hemoglobin level, creatinine level, arterial blood $\mathrm{pH}$ value, and oxygen saturation ( $>0.05$; Table 1). However, in patients in the exacerbation period, the rates of spirometric measurement, mMRC dyspnea scale, and Combined Assessment of COPD score were determined to be significantly higher $(\mathrm{p}<0.01,0.022,0.014$, respectively; Table 2).

\section{PRG4 results}

PRG4 levels of patients in the exacerbation period $(183.7 \pm 9.4$ $\mathrm{ng} / \mathrm{ml}$ ) were significantly higher than those of the patients in the stable period $(177.1 \pm 15.9 \mathrm{ng} / \mathrm{ml} ; \mathrm{p}=0.034$; Table 1$)$. No significant correlation was determined between PRG4 levels and smoking, spirometric measurement, mMRC dyspnea scale, and combined assessment of COPD score (Table 3). In addition, no correlation was found between PRG4 level and PCT and CRP levels, WBC count, and CAT score ( $>0.05$; Table 4).

\section{Other laboratory results}

CRP levels of patients in the exacerbation period $(39.7 \pm 9.1$ $\mathrm{mg} / \mathrm{L}$ ) were significantly higher than those in the patients in the stable period $(7.8 \pm 3.4 \mathrm{mg} / \mathrm{L} ; \mathrm{p}=0.034$; Table 1$)$. However, there was no significant difference between PCT level and WBC count between patients in the exacerbation and stable periods $(\mathrm{p}=0.073$ and 0.139 , respectively; Table 1$)$.

A positive correlations were found between PCT and CRP levels ( $p=0.014$; Table 4). A positive correlations were found between CRP levels and WBC count $(p=0.022$; Table 4). A positive correlations were found between CRP levels and CAT score $(p=0.006$; Table 4).

\section{Cut-off value of PRG4 and other parameters}

For a PRG4 cut-off value of $175.2 \mathrm{ng} / \mathrm{ml}$, sensitivity was calculated as $88.9 \%$ and specificity as $50 \%$. For a PCT cut-off value of $0.031 \mathrm{ng} / \mathrm{ml}$, sensitivity was $88.9 \%$ and specificity was $33.3 \%$. For a CRP cut-off value of $3.45 \mathrm{mg} / \mathrm{L}$, sensitivity was $86.1 \%$ and specificity was $43 \%$. For a WBC cut-off value of $8045 / \mathrm{mm}^{3}$, sensitivity was $80.6 \%$ and specificity was $40 \%$ (Table 5).

Table 2. Comparison of COPD classification scores between exacerbation and stable periods.

\begin{tabular}{|c|c|c|c|}
\hline Classification & $\operatorname{EP}(n=38) n(\%)$ & SP $(n=40) n(\%)$ & $\mathbf{P}$ \\
\hline \multicolumn{4}{|c|}{ Spirometric measurement (GOLD stage) } \\
\hline$\bullet$ & $1(2.6)$ & $4(1.0)$ & $<0.01^{*}$ \\
\hline •॥ & $13(34.2)$ & $28(70.0)$ & \\
\hline • III & $11(28.9)$ & $11(27.5)$ & \\
\hline$\cdot$ IV & $13(34.2)$ & $2(0.5)$ & \\
\hline \multicolumn{4}{|c|}{ mMRC dyspnea scale (grade) } \\
\hline$\cdot 1$ & $3(7.9)$ & $4(10.0)$ & $0.022^{*}$ \\
\hline$\cdot 2$ & $13(34.2)$ & $24(60.0)$ & \\
\hline$\cdot 3$ & $18(47.4)$ & $11(27.5)$ & \\
\hline$\cdot 4$ & $4(10.5)$ & $1(2.5)$ & \\
\hline
\end{tabular}


Combined assessment of COPD ( risk category)

\begin{tabular}{|c|c|c|c|}
\hline - A & $2(5.3)$ & $1(2.6)$ & $0.014^{*}$ \\
\hline - B & $5(13.2)$ & 17 (43.6) & \\
\hline - $\mathrm{C}$ & $7(18.4)$ & $5(12.8)$ & \\
\hline • D & $24(63.2)$ & $16(41.0)$ & \\
\hline
\end{tabular}

COPD: Chronic Obstructive Pulmonary Disease; EP: Exacerbation Period; SP: Stable Period; *Student t-test.

Table 3. The relation between PRG4 and clinical scores used in the evaluation of COPD.

\begin{tabular}{|c|c|c|c|}
\hline \multicolumn{2}{|l|}{ Evaluation criteria } & \multirow{2}{*}{$\begin{array}{l}\text { PRG4 }(\mathrm{ng} / \mathrm{ml}) \\
\text { mean } \pm \mathrm{SD}\end{array}$} & \multirow[t]{2}{*}{$\mathbf{P}$} \\
\hline & & & \\
\hline \multirow[t]{2}{*}{ Smoking status } & Yes $(n=66)$ & $182.0 \pm 8.2$ & \\
\hline & No $(n=12)$ & $180.0 \pm 14.2$ & $0.533^{*}$ \\
\hline \multirow{4}{*}{$\begin{array}{l}\text { Spirometer measurement } \\
\text { stage) }\end{array}$} & $1(n=6)$ & $184.1 \pm 8.8$ & $0.300^{\dagger}$ \\
\hline & $2(n=40)$ & $177.8 \pm 16.2$ & \\
\hline & $3(n=17)$ & $181.2 \pm 9.4$ & \\
\hline & $4(n=15)$ & $185.0 \pm 8.5$ & \\
\hline \multirow[t]{4}{*}{ mMRC dyspnea scale (grade) } & $1(n=7)$ & $183.0 \pm 10.8$ & $0.356^{\dagger}$ \\
\hline & $2(n=37)$ & $178.5 \pm 16.1$ & \\
\hline & $3(n=28)$ & $180.6 \pm 10.6$ & \\
\hline & $4(n=6)$ & $189.4 \pm 13.4$ & \\
\hline \multirow{4}{*}{$\begin{array}{l}\text { Combined assessment of COPD (risk } \\
\text { category) }\end{array}$} & $A(n=4)$ & $181.2 \pm 4.5$ & $0.400^{\dagger}$ \\
\hline & $B(n=22)$ & $177.9 \pm 17.9$ & \\
\hline & $C(n=12)$ & $179.9 \pm 11.2$ & \\
\hline & $\mathrm{D}(\mathrm{n}=40)$ & $191.4 \pm 11.3$ & \\
\hline
\end{tabular}

PRG4: Proteoglycan 4; COPD: Chronic Obstructive Pulmonary Disease; GOLD: Global Initiative for Obstructive Lung Disease; mMRC: modified Medical Research Council; SD: Standard Deviation; "Student t-test; † ${ }^{\dagger}$ NOVA.

\section{Discussion}

The results of this study suggest that PRG4 may be a useful biomarker in distinguishing the exacerbation period from the stable period of COPD. In addition, when we compare PRG4 with the other parameters (PCT and CRP level and WBC count), the specificity of PRG4 was found to be higher, whereas its sensitivity was similar to other parameters in the assessment of the exacerbation period. This proves PRG4 to be superior compared with other parameters in the diagnosis of the exacerbation period of COPD. Literature review revealed that several laboratory parameters (CRP, PCT, nitric oxide, galectin-3, L-arginine, changes in leukocyte count or formula, etc.) have been investigated in order to distinguish the exacerbation and stable periods of COPD [8-14]. However, there are only few studies investigating the relationship between PRG4 and COPD, and the results show that PRG4 levels increase in patients with COPD $[15,16]$. Lee et al. found an increase in PRG4 levels in patients with COPD as the disease progressed and reported that this was due to overstressing of respiratory muscles and impairment of lung compensation because of long-term use of corticosteroids for treatment [7]. Frevert et al. reported that proteoglycans released from the respiratory tract epithelium provided both biomechanical and structural support for the lung tissue and showed that PRG4 levels increased in lung infections [17]. Another study by Lee et al. demonstrated that PRG4 levels in patients with COPD were significantly higher in smokers compared with non-smokers [18].

Table 4. The relationship between CAT scores and laboratory parameters.

\begin{tabular}{lllllll}
\hline Laboratory parameters & PRG4 & PCT & CRP & WBC & CAT score \\
\hline PRG4 & $\mathrm{r}$ & 1 & 0.002 & 0.063 & 0.131 & 0.083 \\
\cline { 2 - 6 } & $\mathrm{p}$ & & 0.984 & 0.583 & 0.252 & 0.47 \\
\hline PCT & $\mathrm{r}$ & 1 & 0.301 & 0.118 & 0.226 \\
\cline { 2 - 6 } & $\mathrm{p}$ & & 0.014 & 0.346 & 0.068 \\
\hline CRP & $\mathrm{r}$ & & 1 & 0.259 & 0.309 \\
\cline { 2 - 6 } & $\mathrm{p}$ & & & 0.022 & 0.006 \\
\hline WBC & $\mathrm{r}$ & & & 1 & 0.067 \\
\cline { 2 - 6 } & $\mathrm{p}$ & & & & 0.56 \\
\hline CAT score & $\mathrm{r}$ & & & & & 1 \\
\cline { 2 - 6 } & $\mathrm{p}$ & & & & & \\
\end{tabular}

PRG4: Proteoglycan 4; PCT: Procalcitonin; CRP: C-Reactive Protein; WBC: White Blood Cell; COPD: Chronic Obstructive Pulmonary Disease; CAT: COPD Assessment Test; r: Spearman's rho correlation coefficient.

In studies investigating the effects of growth factors such as transforming growth factor- $\beta 1$ (TGF- $\beta 1$ ) in patients with COPD, the effect of TGF- $\beta 1$ on cytokine regulation was associated with PRG4, thereby leading to the conclusion that TGF- $\beta 1$ and indirectly PRG4 played a protective role in patients with COPD [19-21]. In their study investigating the effect of proteoglycans on the hematopoietic system, Novince et al. showed that proteoglycans could stimulate hematopoiesis via mRNA in the bone marrow [22]. These results suggest that the damage pattern caused in the lung tissue may be associated with PRG4 and that PRG4 may be a biomarker that indicates lung inflammation and damage. In our study, PRG4 levels in patients in the exacerbation period were higher than those in 
the patients in the stable period, and this increase was statistically significant. Respiratory tract infections are the leading cause of COPD exacerbation [1,2]. The reason for the higher levels of PRG4 in patients in the exacerbation period may be direct damage due to respiratory tract infections in the lungs or damage to the lung tissue due to reasons such as coughing and respiratory distress or excessive stress on the respiratory muscles. In addition, the aggravation of chronic inflammation in patients in the exacerbation period may be considered to be another reason for the increase in PRG4 levels in order to induce hematopoiesis in the bone marrow.

Table 5. Analysis of the laboratory parameters.

\begin{tabular}{|c|c|c|c|c|c|c|}
\hline \multirow[t]{2}{*}{ Parameters } & \multirow[t]{2}{*}{ Area } & \multirow[t]{2}{*}{ Cut-off } & \multirow[t]{2}{*}{ Sensitivity } & \multirow[t]{2}{*}{ Specificity } & \multicolumn{2}{|c|}{ Asymptotic $95 \% \mathrm{Cl}$} \\
\hline & & & & & Lower bound & Upper bound \\
\hline PRG4 (ng/ml) & 0.627 & 175.2 & 88.9 & 50 & 0.485 & 0.769 \\
\hline PCT (ng/ml) & 0.629 & 0.031 & 88.9 & 33.3 & 0.494 & 0.764 \\
\hline $\mathrm{CRP}(\mathrm{mg} / \mathrm{l})$ & 0.65 & 3.45 & 86.1 & 43 & 0.515 & 0.784 \\
\hline WBC $\left(\mathrm{mm}^{3}\right)$ & 0.62 & 8045 & 80.6 & 40 & 0.484 & 0.757 \\
\hline
\end{tabular}

PRG4: Proteoglycan-4; PCT: Procalcitonin; CRP: C-Reactive Protein; WBC: White Blood Cell; COPD: Chronic Obstructive Pulmonary Disease; Cl: Confidence of Interval.

Previous studies have shown that PRG4 levels are higher in patients with COPD who are smokers than in those who are non-smokers [7,18]. The results of this study support this conclusion. However, this increase, which was identified in smokers, was not statistically significant. When we look at the mMRC dyspnea scale that evaluates the dyspnea level in patients with COPD, the Combined Assessment of COPD risk category used in clinical and risk assessment, and PRG4 levels measured in the spirometric evaluation methods used in the diagnosis and management of the disease, we found that although the highest PRG4 levels were measured in stages with the highest severity or the worst clinical impact of the disease (mMRC grade: 4, Spirometric GOLD stage: 4) or higher mortality/morbidity risk (Combined assessment of COPD risk category: D), the difference was not significant when these stages were compared among themselves with respect to their PRG4 levels. In a previous study by Lee et al. a similar result was obtained where PRG4 levels in patients in stages 3 and 4 were found to be higher than those in stages 1 and 2 [7]. Although this result, namely the increase in PRG4 levels in the later stages of the disease, suggests that measuring serum PRG4 levels may be instructive for clinical follow-up and identification of risk category of patients with COPD, the inconsistency between PRG4 levels in patients in the mild and severe stages in different classifications through which diagnosis, follow-up, and risk assessment of COPD is made leads us to question this notion. Nevertheless, further studies are required that investigate the effect of PRG4 levels on clinical follow-up and mortality risk.

When we analyse other biochemical parameters used in our study to differentiate between stable and exacerbation periods of patients with COPD, while a significant increase was found in CRP levels during the exacerbation period, there was no significant difference between PCT level and WBC count in both periods. CRP is a positive acute-phase reactant and is one of the tests used to indicate inflammation (infectious or noninfectious) [2,8-10]. There may be a slight increase in basal
CRP levels in patients with COPD due to increased inflammation over time. However, studies have shown that CRP levels also increase in patients during the exacerbation period because of the severity of the disease [2,23,24]. PintoPlata et al. reported that serum CRP levels were higher in patients with COPD than in those in the control group [10]. In our study, there was a significant increase in CRP levels in patients in the exacerbation period, which was consistent with the literature. This increase may be due to infectious or noninfectious causes, in addition to the gradual increase in baseline CRP levels in patients with COPD. However, the fact that the increase in CRP level in patients in the exacerbation period is correlated with CAT score, which assesses daily activity in patients with COPD, suggests that a possible infectious cause may be also related.

PCT, especially in the presence of bacterial infection, can be released from the lungs, liver, and intestines [25]. Although it is recommended that PCT is studied to clarify the etiology of the disease and the treatment choice during the exacerbation period of COPD, the gradual bacterial colonization that occurs in COPD renders it difficult to use PCT in the diagnosis [25-28]. Flattet et al. reported that PCT levels were not significantly elevated during the exacerbation period of COPD, whereas Civelek et al. found that PCT levels were particularly high in exacerbations caused by infections $[11,26]$. Chang et al., on the other hand, did not find a relationship between PCT level and exacerbation period [8]. In our study, while PCT levels measured in patients in the exacerbation period were higher than those in the patients in the stable period, no statistical difference was found between the two groups. The reason for this can be interpreted as the absence of statistical difference between PCT levels in the two groups due to the lack of elevation of PCT levels associated with viral pathogens or non-infectious causes in some of the patients in the exacerbation period, which also reduces the average PCT level calculated during the exacerbation period, or the increase in PCT levels caused by the gradual increase in bacterial 
colonization in patients in the stable period, which in turn increases the average PCT level calculated in the stable period.

WBC count is another indicator of infection, and its level in the blood increases because of cytokines such as IL-1 and IL-8 that are released by macrophages [2]. No consensus has been reached till date in studies regarding the diagnostic value of WBC count in the exacerbation period of COPD. While two studies reported that the WBC count increased because of systemic inflammation during the exacerbation period and this increase was proportional to the severity of exacerbation $[29,30]$. Chang et al. stated that there was no relationship between the WBC count and exacerbation period [8]. Civelek et al. reported that the increase in the WBC count during the exacerbation period was not statistically significant [11]. In our study, there was no significant difference in the WBC count between patients in the exacerbation period and those in the stable period. We consider that this result is because of the non-microbial causes in the exacerbation period or the lack of an adequate immune response in some infected individuals (immunosuppression, steroids or other drugs use, etc.).

\section{Limitations}

There are certain limitations of our study. First, because the number of patients with COPD during the exacerbation period who present to our Emergency Department particularly increase in the winter season, we tried to select patients who are similar to those in the stable period with respect to age and gender during this limited period. This prevented us from including a larger patient sample. Second, because this is a preliminary study, changes in PRG4 levels and other laboratory parameters of patients during or after treatment, especially in patients admitted during the acute exacerbation period, were not evaluated. Last, the causes that may account for changes in PRG4 levels in our study were identified by considering the detailed medical history of the patients and the records obtained from the hospital information system; moreover, the exclusion criteria were not scientifically proven.

\section{Conclusion}

Our study is a preliminary study and our results show that PRG4 can be a useful biomarker in distinguishing between the exacerbation and stable periods of COPD. In addition, PRG4 appears to be a more specific biomarker that indicates the exacerbation period compared with other biochemical parameters we examined. However, the results obtained in this study were preliminary; therefore, further studies are required for a better understanding of the role of PRG4 in patients with COPD.

\section{Financial Support}

This study was supported by the Scientific Research Project Support Co-ordination Unit of Kirikkale University (No: 2016/078).

\section{References}

1. Vogelmeier CF, Criner GJ, Martinez FJ, Anzueto A, Barnes PJ, Bourbeau J. Global strategy for the diagnosis, management and prevention of chronic obstructive lung disease 2017 report. GOLD executive summary. Respirology 2017; 22: 575-601.

2. Gocmen H, Hikmet C, Yildiz A, Ursavas A, Coskun F, Ediger D. Is there any correlation between serum CRP level and haematological parameters with severity of disease in acute exacerbation of COPD? Respir Dis J 2007; 18: 141-147.

3. Celli BR, MacNee W, Agusti A, Anzueto A, Berg B, Buist AS. Standards for the diagnosis and treatment of patients with COPD: a summary of the ATS/ERS position paper. Eur Respir J 2004; 23: 932-946.

4. Thomsen M, Ingebrigtsen TS, Marott JL, Dahl M, Lange P, Vestbo J, Nordestgaard BG. Inflammatory biomarkers and exacerbations in chronic obstructive pulmonary disease. JAMA 2013; 309: 2353-2361.

5. Iozzo RV, Schaefer L. Proteoglycan form and function: A comprehensive nomenclature of proteoglycans. Matrix Biol 2015; 42: 11-55.

6. Abusara Z, Krawetz R, Steele B, DuVall M, Schmidt T, Herzog W. Muscular loading of joints triggers cellular secretion of PRG4 into the joint fluid. J Biomech 2013; 46: $1225-1230$.

7. Lee KY, Chuang HC, Chen TT, Liu WT, Su CL, Feng PH. Proteoglycan 4 is a diagnostic biomarker for COPD. Int $\mathrm{J}$ Chron Obstruct Pulmon Dis 2015; 10: 1999-2007.

8. Chang $\mathrm{CH}$, Tsao $\mathrm{KC}, \mathrm{Hu} \mathrm{HC}$, Huang CC, Kao KC, Chen NH, Yang CT, Tsai YH, Hsieh MJ. Procalcitonin and Creactive protein cannot differentiate bacterial or viral infection in COPD exacerbation requiring emergency department visits. Int J Chron Obstruct Pulmon Dis 2015; 10: 767-774.

9. Broekhuizen R, Wouters EF, Creutzberg EC, Schols AM. Raised CRP levels mark metabolic and functional impairment in advanced COPD. Thorax 2006; 61: 17-22.

10. Pinto-Plata VM, Mullerova H, Toso JF, Feudjo-Tepie M, Soriano JB, Vessey RS. C-reactive protein in patients with COPD, control smokers and non-smokers. Thorax 2006; 61: 23-28.

11. Civelek C, Korkmaz I, Eren SH, Guven FMK, Karadayi S, Dogan OT. Procalcitonin and other acute phase reactants in patients with chronic obstructive pulmonary disease exacerbation. Dicle Med J 2011; 38: 137-142.

12. Feng W, Wu X, Li S, Zhai C, Wang J, Shi W. Association of serum galectin-3 with the acute exacerbation of chronic obstructive pulmonary disease. Med Sci Monit 2017; 23: 4612-4618.

13. Ruzsics I, Nagy L, Keki S, Sarosi V, Illes B, Illes Z. LArginine pathway in COPD patients with acute exacerbation: a new potential biomarker. COPD 2016; 13 : 139-145. 
14. Malerba M, Radaeli A, Olivini A, Damiani G, Ragnoli B, Montuschi P. Exhaled nitric oxide as a biomarker in COPD and related comorbidities. Biomed Res Int 2014; 2014: 271918.

15. Sato Y, Tanino Y, Wang X, Nikaido T, Sato S, Misa K. Baseline serum syndecan-4 predicts prognosis after the onset of acute exacerbation of idiopathic interstitial pneumonia. Plos One 2017; 12: 0176789.

16. Hallgren $\mathrm{O}$, Nihlberg $\mathrm{K}$, Dahlback $\mathrm{M}$, Bjermer $\mathrm{L}$, Eriksson LT, Erjefalt JS, Lofdahl CG, WestergrenThorsson G. Altered fibroblast proteoglycan production in COPD. Respir Res 2010; 11: 55.

17. Frevert CW, Sannes PL. Matrix proteoglycans as effector molecules for epithelial cell function. Eur Respir Rev 2005; 14: 137-144.

18. Lee KY, Feng PH, Ho SC, Chuang KJ, Chen TT, Su CL. Inter-alpha-trypsin inhibitor heavy chain 4: a novel biomarker for environmental exposure to particulate air pollution in patients with chronic obstructive pulmonary disease. Int J Chron Obstruct Pulmon Dis 2015; 10: 831-841.

19. Konigshoff M, Kneidinger N, Eickelberg O. TGF-beta signaling in COPD: deciphering genetic and cellular susceptibilities for future therapeutic regimen. Swiss Med Wkly 2009; 139: 554-563.

20. Sufaru IG, Beikircher G, Weinhaeusel A, Gruber R. Inhibitors of DNA methylation support TGF-beta1induced IL11 expression in gingival fibroblasts. J Periodontal Implant Sci 2017; 47: 66-76.

21. Cuellar A, Reddi AH. Stimulation of superficial zone protein/lubricin/PRG4 by transforming growth factor- $\beta$ in superficial zone articular chondrocytes and modulation by glycosaminoglycans. Tissue Eng Part A 2015; 21: 1973-1981.

22. Novince CM, Koh AJ, Michalski MN, Marchesan JT, Wang J, Jung Y. Proteoglycan 4, a novel immunomodulatory factor, regulates parathyroid hormone actions on hematopoietic cells. Am J Patho 2011; 179: 2431-2442.

23. Broekhuizen R, Wouters EF, Creutzberg EC, Schols AM. Raised CRP levels mark metabolic and functional impairment in advanced COPD. Thorax 2006; 61: 17-22.
24. Tan DBA, Armitage J, Teo TH, Ong NE, Shin H, Moodley YP. Elevated levels of circulating exosome in COPD patients are associated with systemic inflammation. Respir Med 2017.

25. Biju PG, Garg S, Wang W, Choudhry MA, Kovacs EJ, Fink LM. Procalcitonin as a predictive biomarker for total body irradiation induced bacterial load and lethality in mice. Shock 2012; 38: 170-176.

26. Flattet Y, Garin N, Serratrice J, Perrier A, Stirnemann J, Carballo S. Determining prognosis in acute exacerbation of COPD. Int J Chron Obstruct Pulmon Dis 2017; 12: 467-475.

27. Stolz D, Christ-Crain M, Bingisser R, Leuppi J, Miedinger D, Muller C. Antibiotic treatment of exacerbations of COPD: a randomized, controlled trial comparing procalcitonin-guidance with standard therapy. Chest 2007; 131: 9-19.

28. Desai H, Eschberger K, Wrona C, Grove L, Agrawal A, Grant B. Bacterial colonization increases daily symptoms in patients with chronic obstructive pulmonary disease. Ann Am Thor Soc 2014; 11: 303-309.

29. Sin DD, Man SP. Why are patients with chronic obstructive pulmonary disease at increased risk of cardiovascular diseases? Circulation 2003; 107: 1514-1519.

30. Gan WQ, Man S, Senthilselvan A, Sin D. Association between chronic obstructive pulmonary disease and systemic inflammation: a systematic review and a metaanalysis. Thorax 2004; 59: 574-580.

\section{*Correspondence to}

Oguz Eroglu

Department of Emergency Medicine

Kirikkale University

Turkey 